\title{
Introducing PHY-Layer Fairness in Amplify and Forward Cooperative Diversity Systems
}

\author{
Diomidis S. Michalopoulos, George K. Karagiannidis and Theodoros A. Tsiftsis \\ Department of Electrical and Computer Engineering, \\ Aristotle University of Thessaloniki, Thessaloniki, Greece \\ Email: \{dmixalo, geokarag, thtsif\}@ auth.gr
}

\begin{abstract}
We introduce the concept of fairness at the physicallayer in amplify and forward cooperative diversity systems, which reflects the need for equally allocating the consumed power among the relays. To this end, we propose a relay-selection method, which utilizes knowledge on both the instantaneous and average channel conditions in order to encompass this concept. We also provide a closed-form expression for the outage probability in the high signal to noise ratio (SNR) regime. Numerical results show that the outage performance of the proposed scheme resembles closely the performance of the "best relay selection" scheme, albeit maintaining the average power consumptions equal.
\end{abstract}

\section{INTRODUCTION}

Cooperative diversity (or users cooperating diversity) has recently become an attractive field for researchers devoted to several areas of research [1]- [9], due to the variety of subjects that encompasses regarding the different levels of the Open System Interconnection (OSI) model. Based upon the information sharing among the users, it represents an effective solution for future cellular, wireless LAN or ad-hoc wireless communications systems, promising higher data-rate coverage and better quality of service, without the need for high transmitting power. The cooperating terminals (which are sometimes called partners [4]) create spatial diversity for each other, and thus the cooperative scheme constitutes a virtual antenna array, hence exploiting all the benefits that multiple transmission/reception entails.

Despite these benefits, however, obligating the existing users of a network to serve as the elements of the virtual antenna array as mentioned above, creates a number of newly-appeared problems regarding the efficiency of this scheme in terms of energy and bandwidth usage. To be more precise, since these virtual array elements are not co-located, do not share the same energy deposit and do not correspond to the same traffic generators, protocols that take into consideration the terminals' ability and willingness to act as relays, need to be given attention. In addition, due to the fact that cooperative diversity involves joint transmission of the information signal generated from a single user, in order to enhance the Quality of Service (QoS) of this specific user, it goes without saying that it also involves usage of the resources corresponding to the remaining cooperating users, which they involuntarily waste for the sake of a partner. Therefore, it becomes clear that users cooperating diversity needs also to be studied and thereby designed from a fairness point of view. Regarding the physical layer (PHYlayer), the concept of fairly dealing with the potential relays is in some way analogous with requiring from them equal amount of energy to consume. To this end, the main scope of this work is to introduce the concept of fairness at the PHYlayer in amplify and forward cooperative diversity systems, and thereafter to provide a method of equally allocating the total consumed energy among the relays.

\section{A. Related Work and Results}

The concept of selection cooperation has been recently proposed as a less complex substitute of Distributed Space-Time Coding (DSTC) methods, as it is shown in [5]. Based upon local measurements of the instantaneous channel conditions, the authors developed a distributed method of selecting the best relay from a set of, e.g., $L$ available ones, resulting in diversity gain on the order of $L+1$. Similar results were also derived in [6], where the authors showed that selection cooperation outperforms the DSTC system proposed in [3] in terms of outage probability, albeit simpler.

Motivated by these works, in this paper we propose a method for implementing the concept of PHY-layer fairness, by attributing a weight coefficient to each branch depending on its average fading state, and then selecting the terminal corresponding to the best instantaneous "weighted" channel conditions, in a fashion that ensures equal amount of average consumed power. We also provide a closed-form expression for the outage probability of the proposed scheme in the high signal-to-noise ratio (SNR) regime.

\section{Cooperative Setup}

The system under consideration forms the typical cooperative diversity setup, where a set of $L$ relays are available for forwarding the information signal received from the source node to the destination, each one employing a single transmit/receive antenna. Also, the system is considered centralized; in particular, a central unit (CU) exists (which could be e.g., the source terminal), which knows the average channel conditions of all system links. The source, destination and relay terminals are represented respectively by $S, D$, and $R_{i}$, with $i \in\{1, \ldots, L\}$. All relays are considered to operate in the non-regenerative mode, i.e. they amplify and forward the information signal without any further process. The relaying takes place in a time-orthogonal fashion, which was originally 
proposed in [2]; according to this protocol, each transmission period is divided in two timeslots, corresponding to the $S-R_{i}$ and $R_{i}-D$ communication interval, respectively. Then, at the end of the second timeslot, the destination terminal combines the signal received directly from the source and that received via the relay (which also contains a packet with $S-R_{i}$ channel state information (CSI)), using a maximal ratio combiner (MRC).

Let us denote with $P_{S}$ the power transmitted by the source ${ }^{1}$. Further, we assume that the gain that each relay employs is that proposed in [1], where the relays actually invert the degradation induced by the corresponding $S-R_{i}$ link in order to maintain their output power limited, i.e.,

$$
G_{i}^{2}(t)=\frac{P_{S}}{a_{S i}^{2}(t) P_{S}+N_{0}},
$$

where $a_{S i}(t)$ and $N_{0}$ denote the instantaneous channel gain of the $S-R_{i}$ link and the additive white Gaussian noise (AWGN) power (which is assumed identical throughout the system), respectively. Note that the numerator in (1) implies that all relays transmit with power $P_{S}$ as well. An upper bound of the $S-D$ end-to-end SNR associated with the $i$ th relay is [7]- [9]

$$
\gamma_{i}=\frac{\gamma_{A_{i}} \gamma_{B_{i}}}{\gamma_{A_{i}}+\gamma_{B_{i}}}
$$

where $\gamma_{A_{i}}$ and $\gamma_{B_{i}}$ represent the instantaneous SNRs corresponding to the $S-R_{i}$, and $R_{i}-D$ channel respectively, which in fact corresponds to an ideal relay gain capable of inverting the fading attenuation at the $S-R_{i}$ link ignoring the noise, i.e.,

$$
G_{i}^{2}(t)=\frac{1}{a_{S i}^{2}(t)}
$$

The fading in all channels is assumed to be ergodic, Rayleigh distributed (not necessarily identical), independent with one another. Moreover, all channels are assumed to be quasi-static, that is the fading in each channel remains constant during a coherence time interval and changes independently from one interval to another.

\section{SELECTION IMPLEMENTATION}

The proposed relay selection method encompasses the following two fundamental features:

- utilizing CSI regarding the instantaneous channel gains of the $S-R_{i}, R_{i}-D$ links, the selection is repeated in a rate ensuring constant fading conditions during each transmission period (i.e., this rate is lower than or equal to the inverse of the channel coherence time $T_{c}$ ).

- assuming that the fading statistics of each $S-R_{i}, R_{i}-D$ channel are also known to the $\mathrm{CU}$ before the communication starts, the relay activated for each transmission period actually leads to equal relay power consumption, in a long-term perspective.

\footnotetext{
${ }^{1}$ Without loss of generality, we assume constant $P_{S}$, although this model is also applied when non-binary amplitude modulations are used; in such case, $P_{S}$ represents the average transmitting power over the variable bit amplitudes.
}

In order to satisfy these requirements, for each available relaying terminal $R_{i}, i \in\{1, \ldots, L\}$ we define

$$
b_{i} \triangleq \frac{\gamma_{i}}{\mu_{i}}
$$

where $\mu_{i}$ is a positive constant associated with $R_{i}$. In fact, the $b_{i}$ 's represent the selection metrics in the proposed selection method, allowing for the terminal with the highest $b_{i}$ to relay during the current transmission period, i.e., the node $R_{\kappa}$ is selected to forward the information signal if

$$
\kappa=\arg \max _{i=1, \ldots, L} b_{i}
$$

The selection is assumed to be implemented as follows: at the beginning of the communication session, the $\mu_{i}$ 's are sent to the relays by the $\mathrm{CU}$; then, similarly to the selection method presented in [5], the relays decide in a distributed fashion which one would forward the information for the current interval according to their selection metrics, without the need for any CSI at the source.

Note that the $\mu_{i}$ 's in (4) play the most important role in attaining the desired PHY-layer fairness, since they represent a weight coefficient attributed to each relay in order to control its average selection time along with the corresponding power consumption. As a result, they directly affect the statistics of the $b_{i}$ 's, resulting in a probability density function (PDF) and a cumulative density function (CDF) having the form of (see [7] for the PDF and CDF of $\gamma_{i}$ )

$$
\begin{aligned}
f_{b_{i}}(b)= & \frac{4 b \mu_{i}}{\rho_{i}} e^{-\frac{b \mu_{i} \sigma_{i}}{\rho_{i}}} K_{0}\left(\frac{2 b \mu_{i}}{\sqrt{\rho_{i}}}\right) \\
& +\frac{2 b \mu_{i} \sigma_{i}}{\rho_{i} \sqrt{\rho_{i}}} e^{-\frac{b \mu_{i} \sigma_{i}}{\rho_{i}}} K_{1}\left(\frac{2 b \mu_{i}}{\sqrt{\rho_{i}}}\right)
\end{aligned}
$$

and

$$
F_{b_{i}}(b)=1-\frac{2 b \mu_{i}}{\sqrt{\rho_{i}}} e^{-\frac{b \mu_{i} \sigma_{i}}{\rho_{i}}} K_{1}\left(\frac{2 b \mu_{i}}{\sqrt{\rho_{i}}}\right)
$$

respectively, where $\sigma_{i}=\bar{\gamma}_{A_{i}}+\bar{\gamma}_{B_{i}}, \rho_{i}=\bar{\gamma}_{A_{i}} \bar{\gamma}_{B_{i}}, K_{v}(\cdot)$ represents the modified Bessel function of second kind of order $v$ and the overbar $\left(^{\circ}\right)$ denotes expectation. Also, we denote with $\gamma_{0}$ the direct $S$ - $D$ SNR, and with $\gamma_{e n d}$ the overall output SNR (i.e., $\gamma_{\text {end }}=\gamma_{0}+\gamma_{\kappa}$ ).

\section{A. Determination of the $\mu_{i}$ 's}

Depending upon the average fading conditions only, the $\mu_{i}$ 's are determined at the beginning of the communication session at the CU as we will show later in Section IV$\mathrm{B}$, and remain constant as long as these conditions do not significantly change, in an average sense. Notice that no continuous channel estimation is needed; depending on the propagation environment, the average fading conditions can be estimated using a long training sequence, and continuously improved during the communication period (see also [10]). 


\section{B. Special Cases}

1) Best path selection (BPS): For the special case of $\mu_{1}=$ $\mu_{2}=\ldots=\mu_{L}$, the proposed method reduces to selecting the relay corresponding to the best end-to-end path, which was the case in [5]- [6]. Clearly, such choice for the $\mu_{i}$ 's leads to the maximum attainable performance.

2) Equal selection probability (ESP): If the $\mu_{i}$ 's are set equal to the average end-to-end SNR values corresponding to the $i$ th relay $\left(\mu_{i}=\bar{\gamma}_{i}\right)$, then our model actually "forces" the available terminals to relay for an identical fraction of time. In order to evaluate $\bar{\gamma}_{i}$, we use [11, eq. (3.353.5)], [11, eq. (6.455.1)] and [12] to infer

$$
\begin{aligned}
\bar{\gamma}_{i} & =\frac{1}{\rho_{i}} \int_{0}^{\infty} \int_{0}^{\infty} \frac{x y}{x+y} e^{-x / \bar{\gamma}_{A_{i}}} e^{-y / \bar{\gamma}_{B_{i}}} d x d y \\
& =\frac{\left(1 / \bar{\gamma}_{A_{i}}\right)^{2}-\left(1 / \bar{\gamma}_{B_{i}}\right)^{2}+\left(2 / \rho_{i}\right) \ln \left(\bar{\gamma}_{A_{i}} / \bar{\gamma}_{B_{i}}\right)}{\left(1 / \bar{\gamma}_{A_{i}}-1 / \bar{\gamma}_{B_{i}}\right)^{3}} .
\end{aligned}
$$

Note that the ESP scheme does not necessarily lead to equal amount of consumed power, unless identically distributed fading channels are assumed ${ }^{2}$; in the latter case, the BPS system also coincide with the proposed one.

\section{Attaining the Equal Energy Consumption CASe}

\section{A. Average Relay Power Consumption}

In general, the power consumed by the relays consists of two parts: the power consumed by the tx/rx radio circuitry, including the power needed for signal reception, and the power consumed for amplification. Since the former part is fixed for each relay, our study focuses on the latter one, which naturally comprises the largest part of the total consumption. In the timeorthogonal amplify and forward scenario described in Section II, the relay passes the received signal through an analog delay line and amplifies/retransmits it at the second timeslot. Therefore, the instantaneous power that the $i$ th potential relay consumes can be defined as

- the difference in the instantaneous transmitting and received power when this difference is positive (or equivalently, when $G_{i}>1$ )

- zero, otherwise. This stems from the fact that, when $G_{i} \leq 1$, the resultant signal attenuation can be achieved by utilizing a passive electronic circuit (e.g., a voltage divider).

Denoting the instantaneous transmitting, received and consumed power corresponding to the $i$ th relay by $P_{o_{i}}, P_{i n_{i}}$ and $P_{\text {cons }_{i}}$ respectively, the above statements can be summarized as

$$
P_{\text {cons }_{i}}=\left(P_{o_{i}}-P_{i n_{i}}\right)^{+}=P_{o_{i}}\left(1-\frac{1}{G_{i}^{2}}\right)^{+}
$$

\footnotetext{
${ }^{2}$ Although irrelevant with PHY-layer fairness, selecting the relays with equal probability is important in cross-layer fairness studies, where the concept of fairness is also related to the relays' activation time.
}

where $(\cdot)^{+}=\max (0, \cdot)$. For medium and high SNRs, from (3) and (9) we infer

$$
P_{\text {cons }_{i}}=P_{S}\left(1-a_{S i}^{2}-\frac{N_{0}}{P_{S}}\right)^{+} \approx P_{S}\left(1-\frac{\gamma_{A_{i}}}{S N R}\right)^{+}
$$

where $S N R$ denotes the common SNR without fading, i.e., the ratio

$$
S N R=P_{S} / N_{0}
$$

Let $f_{\gamma_{A_{i}}}^{*}\left(\gamma_{A_{i}}\right)$ denote the conditional PDF of $\gamma_{A_{i}}$, conditioned on the fact that the node $i$ is selected, i.e.,

$$
f_{\gamma_{A_{i}}}^{*}(x)=\frac{f_{\gamma_{A_{i}}}(x) \operatorname{Pr}\left\{i=\kappa \mid \gamma_{A_{i}}\right\}}{\omega_{i}}
$$

where $f_{Z}(\cdot)$ represents the PDF of the random variable (RV) $Z, \omega_{i}$ denotes the unconditional probability that the $i$ th relay is selected and $\operatorname{Pr}\left\{i=\kappa \mid \gamma_{A_{i}}\right\}$ the conditional selection probability, given that $\gamma_{A_{i}}$ is known (i.e., $\gamma_{A_{i}}=x$ ). Then, the average power consumption is given by

$$
\begin{aligned}
P_{i} & =\bar{P}_{\text {cons }_{i}}=\omega_{i} \int_{0}^{\infty} P_{\text {cons }_{i}} f_{\gamma_{A_{i}}}^{*}(x) d x \\
& \approx P_{S} \int_{0}^{S N R}\left(1-\frac{x}{S N R}\right) f_{\gamma_{A_{i}}}(x) \operatorname{Pr}\left\{i=\kappa \mid \gamma_{A_{i}}\right\} d x
\end{aligned}
$$

Since the relay selection is implemented according to (5), the conditional selection probability can be expressed using (2) as

$$
\operatorname{Pr}\left\{i=\kappa \mid \gamma_{A_{i}}\right\}=\int_{0}^{\infty} f_{\gamma_{B_{i}}}(y) \prod_{\substack{j=1 \\ j \neq i}}^{L} F_{b_{j}}\left(\frac{x y}{\mu_{i}(x+y)}\right) d y .
$$

However, considering the complexity of the integral in (14), a closed-form evaluation of $\operatorname{Pr}\left\{i=\kappa \mid \gamma_{A_{i}}\right\}$ with respect to $\mu_{1}, \ldots, \mu_{L}$ seems difficult, if not impossible, especially for large $L$. Alternatively, observing that $b_{i}$ represents the half of the harmonic mean $\mathcal{H}_{\gamma_{A_{i}} / \mu_{i}, \gamma_{B_{i}} / \mu_{i}}$ of $\gamma_{A_{i}} / \mu_{i}, \gamma_{B_{i}} / \mu_{i}$ since

$$
b_{i}=\frac{\gamma_{i}}{\mu_{i}}=\frac{\left(\gamma_{A_{i}} / \mu_{i}\right)\left(\gamma_{B_{i}} / \mu_{i}\right)}{\left(\gamma_{A_{i}} / \mu_{i}\right)+\left(\gamma_{B_{i}} / \mu_{i}\right)},
$$

and using the well-known inequality

$$
\mathcal{H}_{\alpha, \beta}<\min (\alpha, \beta)
$$

for $\alpha, \beta>0$, we can approximate $\operatorname{Pr}\left\{i=\kappa \mid \gamma_{A_{i}}\right\}$ as shown in (A1), with $Y_{Z}(\cdot)$ denoting the complementary cumulative distribution function of the RV $Z$. In other words, instead of selecting the relay with the maximum $b_{i}$, we select that with the highest $\min \left(\gamma_{A_{i}} / \mu_{i}, \gamma_{B_{i}} / \mu_{i}\right)$, which in fact represents an upper bound of $b_{i}$ that is as tight as greater the difference on $\bar{\gamma}_{A_{i}}$ and $\bar{\gamma}_{B_{i}}$ [13].

For simplicity of exposition, let us define

$$
\phi_{j, i} \triangleq \frac{\mu_{j} \sigma_{j}}{\mu_{i} \rho_{j}},
$$

and let $\mathcal{S}_{i}$ represent the set

$$
\mathcal{S}_{i}=\left\{\phi_{j, i}: j, i \in\{1, \ldots, L\}, j \neq i\right\} .
$$




$$
\begin{aligned}
& \operatorname{Pr}\left\{i=\kappa \mid \gamma_{A_{i}}\right\} \approx \operatorname{Pr}\left\{\min \left(\frac{x}{\mu_{i}}, \frac{\gamma_{B_{i}}}{\mu_{i}}\right)>\max _{\substack{j \in\{1, \ldots, L\} \\
j \neq i}} \min \left(\frac{\gamma_{A_{j}}}{\mu_{j}}, \frac{\gamma_{B_{j}}}{\mu_{j}}\right) \mid x\right\} \\
& =\int_{0}^{x} f_{\gamma_{B_{i}}}(y) \prod_{\substack{j=1 \\
j \neq i}}^{L}\left[1-Y_{\gamma_{A_{j}}}\left(\frac{\mu_{j}}{\mu_{i}} y\right) Y_{\gamma_{B_{j}}}\left(\frac{\mu_{j}}{\mu_{i}} y\right)\right] d y+\int_{x}^{\infty} f_{\gamma_{B_{i}}}(y) \prod_{\substack{j=1 \\
j \neq i}}^{L}\left[1-Y_{\gamma_{A_{j}}}\left(\frac{\mu_{j}}{\mu_{i}} x\right) Y_{\gamma_{B_{j}}}\left(\frac{\mu_{j}}{\mu_{i}} x\right)\right] d y \\
& =\frac{1}{\bar{\gamma}_{B_{i}}} \int_{0}^{x} \exp \left(-\frac{y}{\bar{\gamma}_{B_{i}}}\right) \prod_{\substack{j=1 \\
j \neq i}}^{L}\left[1-\exp \left(-\frac{\mu_{j} \sigma_{j}}{\mu_{i} \rho_{j}} y\right)\right] d y+\exp \left(-\frac{x}{\bar{\gamma}_{B_{i}}}\right) \prod_{\substack{j=1 \\
j \neq i}}^{L}\left[1-\exp \left(-\frac{\mu_{j} \sigma_{j}}{\mu_{i} \rho_{j}} x\right)\right]
\end{aligned}
$$

$$
\begin{aligned}
P_{i} & \approx P_{S}\left[1+\frac{\bar{\gamma}_{A_{i}}}{S N R}\left(\exp \left(-\frac{S N R}{\bar{\gamma}_{A_{i}}}\right)-1\right)+\sum_{k=1}^{L-1}(-1)^{k} \sum_{n=1}^{\left(\begin{array}{l}
L-1 \\
k
\end{array}\right)} \frac{1+\frac{\bar{\gamma}_{A_{i}}}{S N R}\left(\exp \left(-S N R / \bar{\gamma}_{A_{i}}\right)-1\right)}{1+\bar{\gamma}_{B_{i}} \sum_{l=1}^{k} \varphi_{l, k, n}^{i}}\right. \\
& \left.+\sum_{k=1}^{L-1}(-1)^{k} \sum_{n=1}^{\left(\begin{array}{c}
L-1 \\
k
\end{array}\right)} \frac{\left[\sigma_{i}+\rho_{i} \sum_{l=1}^{k} \varphi_{l, k, n}^{i}+\frac{\rho_{i}}{S N R}\left(\exp \left(-\frac{\sigma_{i} / \rho_{i}+\sum_{l=1}^{k} \varphi_{l, k, n}^{i}}{1 / S N R}\right)-1\right)\right] \sum_{l=1}^{k} \varphi_{l, k, n}^{i}}{\bar{\gamma}_{A_{i}}\left(\bar{\gamma}_{A_{i}}+\rho_{i} \sum_{l=1}^{k} \varphi_{l, k, n}^{i}\right)\left(\sigma_{i} / \rho_{i}+\sum_{l=1}^{k} \varphi_{l, k, n}^{i}\right)^{2}}\right] .
\end{aligned}
$$

Furthermore, denote by $\mathcal{S}_{k, n}^{i},\left(k=1, \ldots, L, n=1, \ldots,\left(\begin{array}{l}L \\ k\end{array}\right)\right)$ the $n$th $k$-subset of $\mathcal{S}_{i}$, i.e. the $n$th subset of $\mathcal{S}_{i}$ containing exactly $k$ elements; these elements of $\mathcal{S}_{k, n}^{i}$ are denoted with $\varphi_{l, k, n}^{i}(l=1, \ldots, k)$. Then, the second term within the integral of the right-hand side of (A1) can be expressed as

$$
\prod_{\substack{j=1 \\
j \neq i}}^{L}\left[1-e^{-\phi_{j, i} y}\right]=1+\sum_{k=1}^{L-1}(-1)^{k} \sum_{n=1}^{\left(\begin{array}{c}
L-1 \\
k
\end{array}\right)} \exp \left(-y \sum_{l=1}^{k} \varphi_{l, k, n}^{i}\right),
$$

and likewise, substituting $y$ with $x$ in (19) yields an alternative expression for the second term of the right-hand side of (A1). Consequently, from (A1), (13) and after some manipulations, equation (A2) is deduced; combining (A2) and (17), an approximate representation of each $P_{i}$ is derived, with respect to the $\mu_{i}$ 's. For $L=3$, for instance, $P_{1}$ is obtained directly from (A2) by setting $\varphi_{1,1,1}^{1}=\varphi_{1,2,1}^{1}=\mu_{2} \sigma_{2} /\left(\mu_{1} \rho_{2}\right)$ and $\varphi_{1,1,2}^{1}=\varphi_{2,2,1}^{1}=\mu_{3} \sigma_{3} /\left(\mu_{1} \rho_{3}\right)$.

\section{B. Derivation of the desired $\mu_{i}$ 's}

Having found an expression for the $P_{i}$ 's with respect to the $\mu_{i}$ 's, the $\mu_{i}$ 's that lead to equal amount of consumed power are derived by solving the $(L-1) \times(L-1)$ system of simultaneous equations ${ }^{3}$

$$
P_{1}=P_{2}=\ldots=P_{L}
$$

\section{Outage Analysis}

Let $P_{\text {out }}\left(\gamma_{t h}\right)$ denote the outage probability associated with the SNR threshold $\gamma_{t h}$ (i.e., the probability that the received

\footnotetext{
${ }^{3}$ Apparently, it is the relation among the $\mu_{i}$ 's that determines the average consumptions; that is, one of the $\mu_{i}$ 's may take any fixed value (e.g., $\mu_{L}=1$ )
}

SNR falls below $\left.\gamma_{t h}\right)$. It holds

$$
P_{\text {out }}\left(\gamma_{\text {th }}\right)=\sum_{i=1}^{L}\left[\int_{0}^{\gamma_{t h}} f_{g_{i}}(x) \prod_{\substack{j=1 \\ j \neq i}}^{L} F_{b_{j}}\left(\frac{x}{\mu_{i}}\right) d x\right],
$$

where $g_{i}=\gamma_{0}+\gamma_{i}$ (with $g_{\kappa}=\gamma_{\text {end }}$ ) and $f_{g_{i}}(\cdot)$ is the PDF of $g_{i}$, which is actually the convolution of the PDFs of $\gamma_{i}$ and $\gamma_{0}$. Unfortunately, such convolution is not easily tractable and cannot be evaluated in closed form. In the high-SNR region, however, using the approximations $K_{0}(z) \approx-\ln (z)$ and $K_{1}(z) \approx 1 / z$, for $z<<1$ [14, eq. (9.6.8) and (9.6.9)], $f_{g_{i}}(x)$ can be written as

$$
\begin{aligned}
& f_{g_{i}}(x) \\
& =\frac{1}{\bar{\gamma}_{0}} \int_{0}^{x}\left[\frac{\sigma_{i}}{\rho_{i}} e^{-\frac{z \sigma_{i}}{\rho_{i}}}-\frac{4 z}{\rho_{i}} e^{-\frac{z \sigma_{i}}{\rho_{i}}} \ln \left(\frac{2 z}{\sqrt{\rho_{i}}}\right)\right] e^{\frac{1}{\bar{\gamma}_{0}}(z-x)} d z \\
& =\frac{e^{-\frac{x}{\bar{\gamma}_{0}}}}{\bar{\gamma}_{0}}\left[\frac{\sigma_{i}}{\rho_{i}} \frac{1-e^{-\left(\frac{\sigma_{i}}{\rho_{i}}-\frac{1}{\bar{\gamma}_{0}}\right) x}}{\frac{\sigma_{i}}{\rho_{i}}-\frac{1}{\bar{\gamma}_{0}}}-\frac{4}{\rho_{i}} I_{1}\left(\frac{2}{\sqrt{\rho_{i}}}, \frac{\sigma_{i}}{\rho_{i}}-\frac{1}{\bar{\gamma}_{0}}, x\right)\right]
\end{aligned}
$$

where we have introduced the auxiliary function $I_{1}(\cdot, \cdot, \cdot)$ as (please refer to [15])

$$
\begin{aligned}
I_{1}(\delta, b, \omega)= & \int_{0}^{\omega} x \ln (\delta x) e^{-b x} d x \\
= & -\frac{1}{b^{2}}\left[e^{-b \omega}(1+\ln (\delta)+b \omega \ln (\delta))+\Gamma(0, b \omega)\right. \\
& +\ln (\omega) \Gamma(2, b \omega)+\ln (b / \delta)+(u-1)], \quad(23)
\end{aligned}
$$

$\Gamma(\cdot, \cdot)$ denotes the incomplete gamma function defined in [11, eq. (8.350.2)] and $u$ stands for the Euler's constant [11, eq. 


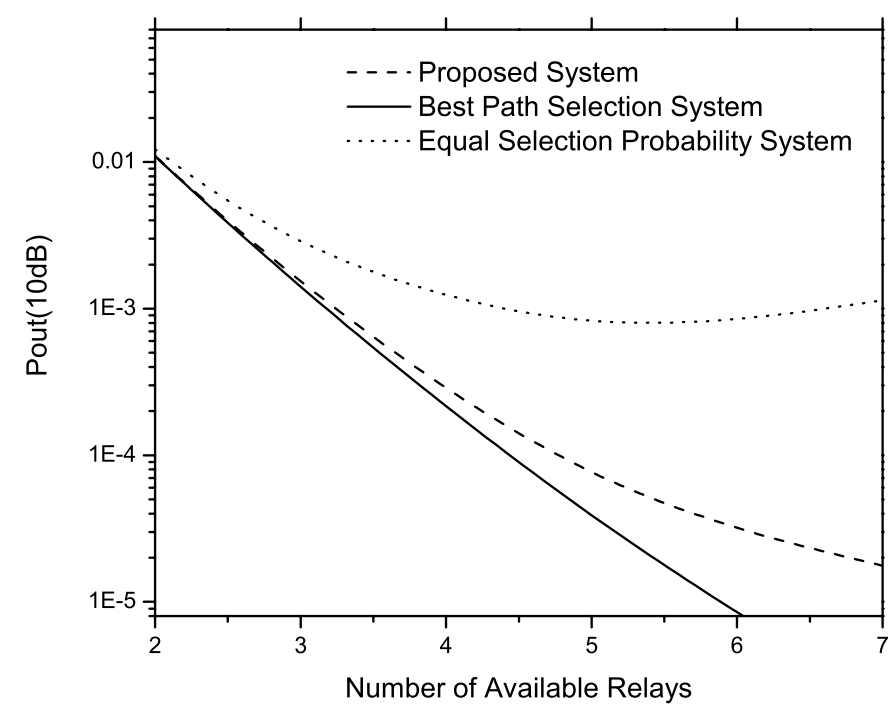

Fig. 1. Outage probability of the proposed, the BPS and the ESP scheme versus the number of available relays, for an SNR threshold equal to 10dB.

(8.367)]. Substituting (22) and (7) into (21), and using again the approximations in $[14$, eq. (9.6.8) and (9.6.9)] we obtain

$$
\begin{aligned}
P_{\text {out }}\left(\gamma_{t h}\right) & =\frac{1}{\bar{\gamma}_{0}} \sum_{i=1}^{L} \int_{0}^{\gamma_{t h}} e^{-\frac{x}{\bar{\gamma}_{0}}}\left[\frac{\sigma_{i}}{\rho_{i}} \frac{1-e^{-\eta_{i} x}}{\eta_{i}}\right. \\
& \left.-\frac{4}{\rho_{i}} I_{1}\left(\frac{2}{\sqrt{\rho_{i}}}, \eta_{i}, x\right)\right] \prod_{\substack{j=1 \\
j \neq i}}^{L}\left[1-e^{-\frac{x \mu_{j} \sigma_{j}}{\mu_{i} \rho_{j}}}\right] d x
\end{aligned}
$$

where we have set

$$
\eta_{i}=\frac{\sigma_{i}}{\rho_{i}}-\frac{1}{\bar{\gamma}_{0}}=\frac{\bar{\gamma}_{A_{i}} \bar{\gamma}_{0}+\bar{\gamma}_{B_{i}} \bar{\gamma}_{0}-\bar{\gamma}_{A_{i}} \bar{\gamma}_{B_{i}}}{\bar{\gamma}_{A_{i}} \bar{\gamma}_{B_{i}} \bar{\gamma}_{0}}
$$

for compactness.

Using (17), (24) can be written as

$$
P_{\text {out }}\left(\gamma_{\text {th }}\right)=\frac{1}{\bar{\gamma}_{0}} \sum_{i=1}^{L}\left[\Psi_{i}+\Omega_{i}\right]
$$

where $\Psi_{i}$ and $\Omega_{i}$ are given in Appendix I, in equations (B1) and (B2), respectively.

\section{NumERICAL EXAMPLES}

In this section we compare the performance of the proposed relay-selection method with that of the BPS and the ESP scheme (Sections III-B.1 and III-B.2 respectively), in terms of outage probability and PHY-layer fairness attainment, when operating over independent Rayleigh fading channels. In our examples, the average SNRs of the $S-R_{i}$ and $R_{i}-D$ links are considered to follow an exponential profile with decay factor $\delta=0.5$ and initial value equal to $26 \mathrm{~dB}$ and $28 \mathrm{~dB}$, respectively (i.e., $\bar{\gamma}_{A_{i}}=10^{2.6} e^{-0.5(i-1)}, \quad \bar{\gamma}_{B_{i}}=10^{2.8} e^{-0.5(i-1)}, i \in$ $\{1, \ldots, L\})$. Fig. 1 depicts the outage probability of the above systems versus the number of available relays for $\gamma_{t h}=$ $10 \mathrm{~dB}$. The main result extracted from this figure is that the performance of the proposed scheme resembles that of the BPS
TABLE I

MeAn of THE AVERAGE POWER CONSUMPTIONS AS A PERCENTAGE OF $P_{S}$ AND STANDARd DEVIATION OVER MEAN OF THE PROPOSED, THE BPS AND THE ESP SCHEME.

\begin{tabular}{|c|c|cc|cc||}
\hline \hline \multicolumn{5}{||c||}{ Mean of the Average Power Consumptions as a percentage of $P_{S}$} \\
and Standard Deviation over Mean \\
\hline \multirow{2}{*}{$L$} & $\begin{array}{c}\text { Prop. } \\
\text { Scheme }\end{array}$ & \multicolumn{2}{|c}{ BPS } & \multicolumn{2}{c||}{ ESP } \\
\hline \multirow{2}{*}{2} & Mean & Mean & SD/Mean & Mean & SD/Mean \\
3 & $6.52 \%$ & $6.51 \%$ & $4.04 \%$ & $6.93 \%$ & $33.52 \%$ \\
5 & $3.61 \%$ & $3.57 \%$ & $12.54 \%$ & $4.59 \%$ & $62.68 \%$ \\
7 & $2.22 \%$ & $2.03 \%$ & $43.23 \%$ & $4.58 \%$ & $89.25 \%$ \\
\hline \hline
\end{tabular}

system for small $L$, and that the difference on performance between these systems increases as $L$ grows large. In other words, selecting the relay according to a PHY-layer fairness rule instead of selecting the one with the best end-to-end SNR does have a cost on performance only for large $L$, since large $L$ implies that many enough "shadowed" terminals are also participating in the relaying process, resulting in performance degradation. Also, notice that both the proposed and the BPS scheme outperform the ESP one.

In Table I, we provide a PHY-layer fairness attainment comparison of the three schemes mentioned above. We use the $P_{i}$ 's standard deviation (SD) over mean as a fairness metric, since it represents the most common order-of-magnitude metric of the unevenness of the energy consumption distribution among the available relays. The mean value of the $P_{i}$ 's is also provided, as a percentage of $P_{S}$, for several values of $L$. The $P_{i}$ 's $\mathrm{SD}$ for the proposed scheme is by definition equal to zero. As we can see from Table I, the proposed scheme's mean value of the average power consumptions is the nearly the same as that of the BPS scheme, and lower than that of the ESP. However, both the BPS and ESP schemes lead to a nonnegligible SD over mean of $P_{i}$ 's, a fact which characterizes them as "non-fair" in terms of energy consumption, especially for large values of $P_{S}$.

\section{APPENDIX I}

The $\Psi_{i}$ and $\Omega_{i}$ in (26) are given by (B1) and (B2) respectively, where the auxiliary functions $I_{2}(\cdot, \cdot), I_{3}(\cdot, \cdot, \cdot)$ and $I_{4}(\cdot, \cdot)$ in $(\mathrm{B} 2)$ are defined as

$$
\begin{aligned}
& I_{2}(b, \omega)=\int_{0}^{\omega} x e^{-b x} d x=\frac{1}{b^{2}}\left[1-e^{-b \omega}(1+b \omega)\right] \\
& I_{3}(\varepsilon, b, \omega)=\int_{0}^{\omega} e^{-b x} \Gamma(0, \varepsilon x) d x \\
& =\frac{\ln (b+\varepsilon)-\ln (\varepsilon)-e^{-b \omega} \Gamma(0, \varepsilon \omega)+\Gamma(0,(b+\varepsilon) \omega)}{b}
\end{aligned}
$$




$$
\Psi_{i}=\frac{\sigma_{i}}{\rho_{i} \eta_{i}}\left[\bar{\gamma}_{0}\left(1-e^{-\frac{\gamma_{t h}}{\gamma_{0}}}\right)-\frac{1-e^{-\frac{\sigma_{i}}{\rho_{i}} \gamma_{t h}}}{\sigma_{i} / \rho_{i}}+\sum_{k=1}^{L-1} \sum_{n=1}^{\left(\begin{array}{c}
L-1 \\
k
\end{array}\right)}\left(\frac{1-e^{-\gamma_{t h}\left(1 / \bar{\gamma}_{0}+\sum_{l=1}^{k} \varphi_{l, k, n}^{i}\right)}}{(-1)^{k}\left(\frac{1}{\bar{\gamma}_{0}}+\sum_{l=1}^{k} \varphi_{l, k, n}^{i}\right)}+\frac{1-e^{-\gamma_{t h}\left(\sigma_{i} / \rho_{i}+\sum_{l=1}^{k} \varphi_{l, k, n}^{i}\right)}}{(-1)^{k}\left(\frac{\sigma_{i}}{\rho_{i}}+\sum_{l=1}^{k} \varphi_{l, k, n}^{i}\right)}\right)\right]
$$

$$
\begin{aligned}
& \Omega_{i}=\frac{4}{\rho_{i} \eta_{i}^{2}}\left[\bar{\gamma}_{0}\left(\ln \left(\frac{\eta_{i} \sqrt{\rho_{i}}}{2}\right)+u-1\right)\left(1-e^{-\gamma_{t h} / \bar{\gamma}_{0}}\right)+\frac{\rho_{i}}{\sigma_{i}}\left(1+\ln \left(\frac{2}{\sqrt{\rho_{i}}}\right)\right) 1-e^{-\gamma_{t h} \frac{\sigma_{i}}{\rho_{i}}}+\eta_{i} \ln \left(\frac{2}{\sqrt{\rho_{i}}}\right) I_{2}\left(\frac{\sigma_{i}}{\rho_{i}}, \gamma_{t h}\right)\right. \\
& +I_{3}\left(\eta_{i}, 1 / \bar{\gamma}_{0}, \gamma_{t h}\right)+I_{4}\left(\eta_{i}, 1 / \bar{\gamma}_{0}, \gamma_{t h}\right)+\left(\ln \left(\frac{\eta_{i} \sqrt{\rho_{i}}}{2}\right)+u-1\right) \sum_{k=1}^{L-1}(-1)^{k} \sum_{n=1}^{\left({ }^{L-1}\right)} \frac{1-\exp \left(-\gamma_{t h}\left(1 / \bar{\gamma}_{0}+\sum_{l=1}^{k} \varphi_{l, k, n}^{i}\right)\right)}{1 / \bar{\gamma}_{0}+\sum_{l=1}^{k} \varphi_{l, k, n}^{i}} \\
& +\left(1+\ln \left(\frac{2}{\sqrt{\rho_{i}}}\right)\right) \sum_{k=1}^{L-1} \sum_{n=1}^{\left(\begin{array}{c}
L-1 \\
k
\end{array}\right)} \frac{1-\exp \left(-\gamma_{t h}\left(\frac{\sigma_{i}}{\rho_{i}}+\sum_{l=1}^{k} \varphi_{l, k, n}^{i}\right)\right)}{(-1)^{k}\left(\sigma_{i} / \rho_{i}+\sum_{l=1}^{k} \varphi_{l, k, n}^{i}\right)}+\eta_{i} \ln \left(\frac{2}{\sqrt{\rho_{i}}}\right) \sum_{k=1}^{L-1} \sum_{n=1}^{\left(\begin{array}{c}
L-1 \\
k
\end{array}\right)} \frac{I_{2}\left(\frac{\sigma_{i}}{\rho_{i}}+\sum_{l=1}^{k} \varphi_{l, k, n}^{i}, \gamma_{t h}\right)}{(-1)^{k}} \\
& \left.+\sum_{k=1}^{L-1}(-1)^{k} \sum_{n=1}^{\left(\begin{array}{c}
L-1 \\
k
\end{array}\right)} I_{3}\left(\eta_{i}, \frac{1}{\bar{\gamma}_{0}}+\sum_{l=1}^{k} \varphi_{l, k, n}^{i}, \gamma_{t h}\right)+\sum_{k=1}^{L-1}(-1)^{k} \sum_{n=1}^{\left(\begin{array}{c}
L-1 \\
k
\end{array}\right)} I_{4}\left(\eta_{i}, \frac{1}{\bar{\gamma}_{0}}+\sum_{l=1}^{k} \varphi_{l, k, n}^{i}, \gamma_{t h}\right)\right]
\end{aligned}
$$

$$
\begin{aligned}
& I_{4}(\varepsilon, b, \omega)=\int_{0}^{\omega} \ln (x) e^{-b x} \Gamma(2, \varepsilon x) d x \\
& =-\frac{\ln (\omega) e^{-(b+\varepsilon) \omega}+\Gamma(0,(b+\varepsilon) \omega)+\ln (b+\varepsilon)+u}{b+\varepsilon} \\
& \quad-\frac{1}{(b+\varepsilon)^{2}}\left[e^{-(b+\varepsilon) \omega}+\Gamma(0,(b+\varepsilon) \omega)\right. \\
& \quad+\ln (\omega) \Gamma(2,(b+\varepsilon) \omega)+\ln (b+\varepsilon)+u-1] .
\end{aligned}
$$

For the analytical derivation of (28) and (29) the reader is referred to [15].

\section{ACKNOWLEDGMENT}

This work was performed within the framework of the project PENED 2003, funded by the European UnionEuropean Social Fund, and from the GSRT of the Hellenic Ministry of Development.

\section{REFERENCES}

[1] J. N. Laneman, D. N. C. Tse, and G. W. Wornell, "Cooperative diversity in wireless networks: Efficient protocols and outage behaviour," IEEE Trans. Inform. Theory, vol. 50, pp. 3062-3080, Dec. 2004.

[2] J. N. Laneman, "Cooperative Diversity in Wireless Networks: Algorithms and Architectures," Ph.D. dissertation, Massachusetts Institute of Technology, Cambridge, MA, Aug. 2002.

[3] J. N. Laneman and G. W. Wornell, "Distributed space-time-coded protocols for exploiting cooperative diversity in wireless networks," IEEE Trans. Inform. Theory, vol. 49, pp. 2415-2425, Nov. 2003.
[4] A. Sendonaris, E. Erkip, and B. Aazhang, "User cooperation diversityPart I: System description," IEEE Trans. Commun., vol. 51, pp. 19271938, Nov. 2003.

[5] A. Bletsas, A. Khisti, D. P. Reed, and A. Lippman, "A simple cooperative diversity method based on network path selection," IEEE J. Selec. Areas Commun., vol. 24, pp. 659-672, Mar. 2006.

[6] E. Beres and R. Adve, "On selection cooperation in distributed networks," in Proc. of Conference on Information Sciences and Systems (CISS 06), Princeton, NJ, Mar. 2006.

[7] P. A. Anghel and M. Kaveh, "Exact symbol error probability of a cooperative network in a Rayleigh-fading environment," IEEE Trans. Wir. Commun., vol. 3, pp. 1416-1421, Sept. 2004.

[8] A. Ribeiro, X. Cai, and G. Giannakis, "Symbol error probabilities for general cooperative links," IEEE Trans. Wir. Commun., vol. 4, pp. 12641273, May 2005.

[9] M. O. Hasna and M. S. Alouini, "End-to-end performance of transmission systems with relays over Rayleigh fading channels," IEEE Trans. Wir. Commun., vol. 2, pp. 1126-1131, Nov. 2003.

[10] R. You, H. Li, and Y. Bar-Ness, "Diversity combining with imperfect channel estimation," IEEE Trans. Commun., vol. 53, pp. 1655-1662, Oct. 2005.

[11] I. S. Gradshteyn and I. M. Ryzhik, Table of Integrals, Series, and Products, 6th ed. New York: Academic, 2000.

[12] Wolfram. (2006) The Wolfram functions site. Internet. [Online]. Available: http://functions.wolfram.com/07.23.03.0428.01

[13] P. S. Bullen, D. S. Mitrinovic, and P. M. Vasic, Means and their Inequalities. Dordrecht: Kluwer Academic Publishers, 1988.

[14] M. Abramovitz and I. A. Stegun, Handbook of Mathematical Functions with Formulas, Graphs, and Mathematical Tables, 9th ed. New York: Dover, 1972.

[15] (2006) Internet. [Online]. http://users.auth.gr/dmixalo/pdf/ICCextra1.pdf 